\title{
The Change of Systemic Immune-Inflammation Index Independently Predicts Survival of Colorectal Cancer Patients after Curative Resection
}

\author{
Qingqing Chen, ${ }^{1}$ Haohao Wu, ${ }^{2}$ Xinwei Guo, ${ }^{3} \mathrm{Ke} \mathrm{Gu},{ }^{4}$ Wenjie Wang, ${ }^{1}$ Xiaochen Chen, ${ }^{1}$ \\ Shengjun Ji $\left(\mathbb{1}^{1},{ }^{1}\right.$ Hui Yang, ${ }^{1}$ and Jiahao Zhu $\mathbb{C}^{4}$ \\ ${ }^{1}$ Department of Radiotherapy \& Oncology, Nanjing Medical University Affiliated Suzhou Hospital, Suzhou, China \\ ${ }^{2}$ Department of Radiotherapy, The Yancheng No. 1 People's Hospital, Yancheng, China \\ ${ }^{3}$ Department of Oncology, Affiliated Taixing People's Hospital of Yangzhou University, Taixing, China \\ ${ }^{4}$ Department of Radiotherapy \& Oncology, Affiliated Hospital of Jiangnan University, Wuxi, China
}

Correspondence should be addressed to Shengjun Ji; happy168123@aliyun.com and Jiahao Zhu; zjhmedicine@163.com

Received 27 June 2020; Revised 12 September 2020; Accepted 27 September 2020; Published 16 November 2020

Academic Editor: Giuseppe Valacchi

Copyright ( 2020 Qingqing Chen et al. This is an open access article distributed under the Creative Commons Attribution License, which permits unrestricted use, distribution, and reproduction in any medium, provided the original work is properly cited.

\begin{abstract}
Background. The systemic immune-inflammation index (SII) has an important role in predicting survival in some solid tumors. However, little information is available concerning the change of the SII $(\Delta$ SII) in colorectal cancer (CRC) after curative resection. This study was designed to evaluate the role of $\Delta$ SII in CRC patients who received surgery. Methods. A total 206 patients were enrolled in this study. Clinicopathologic characteristics and survival were assessed. The relationships between overall survival (OS), disease-free survival (DFS), and $\Delta$ SII were analyzed with both univariate Kaplan-Meier and multivariate Cox regression methods. Results. Based on the patient data, the receiver operating characteristic (ROC) optimal cutoff value of $\Delta$ SII was 127.7 for OS prediction. The 3-year and 5-year OS rates, respectively, were $60.4 \%$ and $36.7 \%$ in the high- $\Delta$ SII group $(>127.7)$ and $87.6 \%$ and $79.8 \%$ in the low- $\Delta$ SII group ( $\leq 127.7)$. The 3-year and 5-year DFS rates, respectively, were $54.1 \%$ and $34.1 \%$ in the high- $\Delta$ SII group and $80.3 \%$ and $78.5 \%$ in the low- $\Delta$ SII group. In the univariate analysis, smoking, pathological stages III-IV, high-middle degree of differentiation, lymphatic invasion, vascular invasion, and the high- $\Delta$ SII group were associated with poor OS. Adjuvant therapy, pathological stages III-IV, vascular invasion, and $\triangle$ SII were able to predict DFS. Multivariate analysis revealed that pathological stages III-IV ( $\mathrm{HR}=0.442,95 \% \mathrm{CI}=0.236-0.827, p=0.011)$, vascular invasion $(\mathrm{HR}=2.182,95 \% \mathrm{CI}=1.243-3.829, p=0.007)$, and the high- $\Delta \mathrm{SII}$ group $(\mathrm{HR}=4.301,95 \% \mathrm{CI}=2.517-7.350, p<0.001)$ were independent predictors for OS. Adjuvant therapy $(\mathrm{HR}=0.415,95 \% \mathrm{CI}=0.250-0.687, p=0.001)$, vascular invasion $(\mathrm{HR}=3.305$, $95 \% \mathrm{CI}=1.944-5.620, p<0.001)$, and the high- $\Delta \mathrm{SII}$ group $(\mathrm{HR}=4.924,95 \% \mathrm{CI}=2.992-8.102, p<0.001)$ were significant prognostic factors for DFS. Conclusions. The present study demonstrated that $\Delta$ SII was associated with the clinical outcome in CRC patients undergoing curative resection, supporting the role of $\Delta$ SII as a prognostic biomarker.
\end{abstract}

\section{Introduction}

Colorectal cancer (CRC) is one of the most common malignant diseases $[1,2]$. The clinical outcome of CRC is still unsatisfactory because of recurrence or metastasis. In addition, despite significant advances in CRC treatment with intense research activity and outcomes, the appropriate stratification of CRC patients remains a challenge. There is still heterogeneity in the prognosis between CRC with the same tumor nodal metastasis (TNM) stage and CRC with the same Dukes' classification $[3,4]$, which indicates that they are not sufficient to correctly stratify patients in terms of the risk of mortality $[5,6]$. Other conventional prognostic biomarkers, such as tumor differentiation and pathological type, have been used for predicting CRC outcomes $[7,8]$. However, these are tumor tissue dependent and the detections are 
usually costly and time-consuming. Therefore, it is very urgent to develop auxiliary biomarkers to help clinicians to identify individualized treatment.

In recent years, a lot of inflammation-based prognostic systems are gradually getting attention $[9,10]$, with the role of inflammation in angiogenesis promotion, DNA damage, and tumor invasion and metastasis [11-13]. Several immune-inflammation systems, such as the neutrophillymphocyte ratio (NLR), platelet-lymphocyte ratio (PLR), and lymphocyte-to-monocyte ratio (LMR), have been reported to predict the prognosis for some malignant solid tumors [14-17]. Systemic immune-inflammation index (SII) is a novel parameter related to the platelet, neutrophil, and lymphocyte. Recently, SII has been reported for its prognostic role in some solid tumors such as liver cancer, nonsmall-cell lung cancer, renal cell cancer, and esophageal squamous cell carcinoma [18-20]. Although many studies also indicated the prognostic role of SII in CRC patients, the results were inconsistent [21-23]. Noteworthy, some studies have found that the dynamic change in the SII represents a new indicator for predicting the prognosis in renal cell cancer [20] and hepatocellular carcinoma [24]. However, the role of the change in SII $(\Delta$ SII) before and after treatment is unknown for patients undergoing curative surgery for CRC. Therefore, we designed the study to evaluate the prognostic value of $\triangle \mathrm{SII}$ in $\mathrm{CRC}$ patients who received curative resection.

\section{Materials and Methods}

2.1. Patients. In total, 206 sequential patients diagnosed with CRC who were treated at our institute from February 2010 to May 2015 were collected. The tumor stage was classified according to the seventh edition of the American Joint Committee on Cancer (AJCC) TNM classification system. The clinicopathological characteristics and laboratory data were collected for each patient. Patients were included if they underwent surgical resection and had sufficient laboratory data and follow-up data available. Patients with infection and emergency cases or patients using anti-inflammatory or immunosuppressive medicines were excluded. We also excluded the patients who received neoadjuvant therapy, chemotherapy, or radiotherapy. Follow-up assessments included routine laboratory and physical examinations as well as imaging examinations every 3 months in the first 3 years and every 6 months thereafter. The end points were overall survival (OS) and disease-free survival (DFS).

2.2. Ethical Statement. All procedures performed were in accordance with the ethical standards of the responsible committee for human experimentation (institutional and national) and with the Helsinki Declaration of 1964 and later versions. This study was approved by the Institutional Review Board of Nanjing Medical University Affiliated Suzhou Hospital (No. KL901056).

2.3. Calculation and Definition of SII and $\Delta$ SII. The platelet, neutrophil, and lymphocyte count at 7-day preoperative and postoperative. The SII was calculated as follows:
TABLE 1: Baseline characteristics.

\begin{tabular}{|c|c|c|}
\hline Variables & $n$ & $\%$ \\
\hline \multicolumn{3}{|l|}{ Age (years) } \\
\hline$>60$ & 73 & 35.4 \\
\hline$\leq 60$ & 133 & 64.6 \\
\hline \multicolumn{3}{|l|}{ Gender } \\
\hline Male & 108 & 52.4 \\
\hline Female & 98 & 47.6 \\
\hline \multicolumn{3}{|l|}{ Smoking status } \\
\hline Yes & 63 & 30.6 \\
\hline No & 143 & 69.4 \\
\hline \multicolumn{3}{|l|}{${ }^{\dagger} \mathrm{KPS}$} \\
\hline$>80$ & 148 & 71.8 \\
\hline$\leq 80$ & 58 & 28.2 \\
\hline \multicolumn{3}{|l|}{ Localization } \\
\hline Rectum & 121 & 58.7 \\
\hline Colon & 85 & 41.3 \\
\hline \multicolumn{3}{|c|}{ Surgical approach } \\
\hline Laparoscopy & 117 & 56.8 \\
\hline Open & 89 & 43.2 \\
\hline \multicolumn{3}{|l|}{ Adjuvant therapy } \\
\hline Yes & 101 & 49.0 \\
\hline No & 105 & 51.0 \\
\hline \multicolumn{3}{|l|}{${ }^{\dagger} \mathrm{CEA}(\mathrm{ng} / \mathrm{ml})$} \\
\hline$>5$ & 89 & 43.2 \\
\hline$\leq 5$ & 117 & 56.8 \\
\hline \multicolumn{3}{|c|}{ Pathological stage } \\
\hline Stages I-II & 109 & 52.9 \\
\hline Stages III-IV & 97 & 47.1 \\
\hline \multicolumn{3}{|c|}{ Differentiated degree } \\
\hline High-middle & 173 & 84.0 \\
\hline Minor & 33 & 16.0 \\
\hline \multicolumn{3}{|c|}{ Lymphatic invasion } \\
\hline Yes & 99 & 48.1 \\
\hline No & 107 & 51.9 \\
\hline \multicolumn{3}{|c|}{ Vascular invasion } \\
\hline Yes & 99 & 48.1 \\
\hline No & 107 & 51.9 \\
\hline
\end{tabular}

${ }^{\dagger}$ KPS: Karnofsky Performance Status; CEA: carcinoembryonic antigen.

SII = platelet counts $*$ neutrophil counts/lymphocyte counts. The $\Delta$ SII (change in SII) was calculated as the postoperative SII minus the preoperative SII.

2.4. Statistical Analysis. Association analysis was performed with the Fisher exact test or the chi-squared test, when appropriate. The Kaplan-Meier method and log-rank test were used for subsistence analysis. OS was the time between surgery and death or the last follow-up. DFS was the time between surgery and the first relapse (local recurrence and/or distant metastases). Univariate and multivariate Cox regression analyses were used to evaluate independent prognostic value. The optimal cutoff value was calculated with receiver 


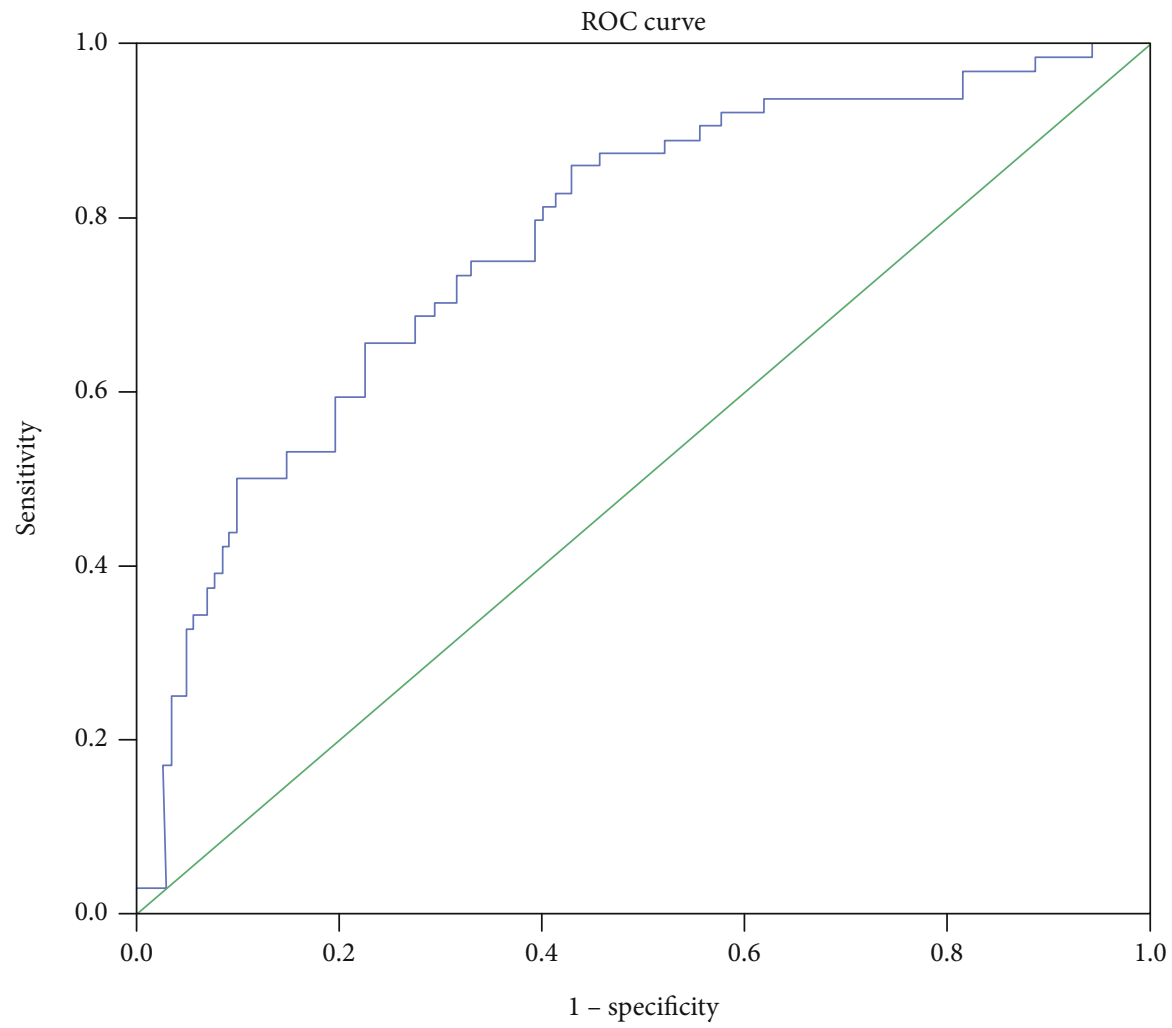

FIGURE 1: Receiver-operating characteristic curves for overall survival prediction.

operating characteristic (ROC) curves. All tests were twosided, and $p<0.05$ was considered to be significant. The SPSS 25.0 statistical software (IBM Corporation, Armonk, NY, USA) was used to statistical analysis.

\section{Results}

3.1. Patient Characteristics. A total of 206 CRC patients received surgical were enrolled in the study. The median age was 56.8 years (27-83 years). The median follow-up duration was 46.5 months (8-98 months). The male and female patients were $108(52.4 \%)$ and 98 (47.6\%), respectively. In total, the number of early-stage (I-II) patients was 109 (52.9\%) and the number of advanced-stage (III-IV) patients was $97(47.1 \%)$. A total of $101(49.0 \%)$ patients received adjuvant therapy (radiation, chemotherapy, or radiochemotherapy). The baseline of patient characteristics is shown in Table 1.

3.2. Survival Results and Prognostic Values. For all patients, before the last follow-up, 64 (31.1\%) patients died, and 74 (35.9\%) patients developed recurrence. The median DFS and OS were 44.6 months (2-80 months) and 51.6 months (8-98 months), respectively. The 3-year and 5-year OS rates were $80.1 \%$ and $34 \%$. The 3 -year and 5 -year DFS rates were $71.4 \%$ and $25.7 \%$ for the entire study population.

3.3. Overall Survival Prediction with ROC Curve. We attempted to select the optimal cutoff for the $\Delta$ SII in our study with ROC curve analysis. The optimal cutoff value for the $\Delta$ SII was127.7 for the OS prediction, with an AUC of 0.774 (sensitivity $=65.6 \%$ and specificity $=77.5 \%$ ) (Figure 1). Consequently, the patients were divided into two groups, the high $\Delta$ SII group $(\Delta \mathrm{SII}>127.7)$ or low $\Delta$ SII group $(\Delta \mathrm{SII} \leq 127.7) .74$ patients $(35.9 \%)$ with high $\Delta$ SII were considered the high-risk group, and 132 patients (64.1\%) with low $\Delta$ SII were considered the low-risk group.

3.4. The $\triangle S I I$ and Clinicopathological Characteristics. The clinicopathological characteristics in the high- $\Delta$ SII group $(>127.7)$ and low- $\Delta$ SII group $(\leq 127.7)$ are shown in Table 2. There were no significant differences between the two groups, except for age $(p<0.001)$, adjuvant therapy $(p=0.016)$, and vascular invasion $(p=0.013)$.

3.5. Overall Survival and Disease-Free Survival according to the $\triangle S I I$. The OS and DFS was estimated with the KaplanMeier method (Figures 2 and 3). The 3-year and 5-year OS rates, respectively, were $60.4 \%$ and $36.7 \%$ in the high- $\Delta$ SII group and $87.6 \%$ and $79.8 \%$ in the low- $\Delta$ SII group. The $3-$ year and 5-year DFS rates, respectively, were $54.1 \%$ and $34.1 \%$ in the high- $\Delta$ SII group and $80.3 \%$ and $78.5 \%$ in the low- $\Delta$ SII group.

3.6. Univariate and Multivariate Cox Regression Survival Analyses. In the univariate analysis, smoking $(\mathrm{HR}=1.890$, $95 \% \mathrm{CI}=1.028-3.476, p=0.040)$, pathological stages III-IV $(\mathrm{HR}=0.451,95 \% \mathrm{CI}=0.270-0.755, p=0.002)$, a highmiddle degree of differentiation $(\mathrm{HR}=0.476,95 \% \mathrm{CI}=$ $0.262-0.862, p=0.014)$, lymphatic invasion $(\mathrm{HR}=1.934$, 
TABLE 2: Clinicopathological variables of 206 colorectal cancer patients according to the $\Delta$ SII.

\begin{tabular}{|c|c|c|c|}
\hline Variables & $\begin{array}{c}{ }^{\dagger} \Delta \text { SII }>127.7 \\
N=74(35.9 \%)\end{array}$ & $\begin{array}{c}\Delta \mathrm{SII} \leq 127.7 \\
N=132(64.1 \%)\end{array}$ & $p$ \\
\hline \multicolumn{4}{|l|}{ Age (years) } \\
\hline$>60$ & $38(51.4)$ & $35(26.5)$ & $<0.001$ \\
\hline$\leq 60$ & $36(48.6)$ & $97(73.5)$ & \\
\hline \multicolumn{4}{|l|}{ Gender } \\
\hline Male & $33(44.6)$ & $75(56.8)$ & 0.092 \\
\hline Female & $41(55.4)$ & $57(43.2)$ & \\
\hline \multicolumn{4}{|l|}{ Smoking status } \\
\hline Yes & $20(27.0)$ & $43(32.6)$ & 0.407 \\
\hline No & $54(73.0)$ & $89(67.4)$ & \\
\hline \multicolumn{4}{|l|}{${ }^{\dagger} \mathrm{KPS}$} \\
\hline$>80$ & $51(68.9)$ & $97(73.5)$ & 0.485 \\
\hline$\leq 80$ & $23(31.1)$ & $35(26.5)$ & \\
\hline \multicolumn{4}{|l|}{ Localization } \\
\hline Rectum & $46(62.2)$ & $75(56.8)$ & 0.455 \\
\hline Colon & $28(37.8)$ & $57(43.2)$ & \\
\hline \multicolumn{4}{|c|}{ Surgical approach } \\
\hline Laparoscopy & $42(56.8)$ & $75(56.8)$ & 0.993 \\
\hline Open & $32(43.2)$ & $57(43.2)$ & \\
\hline \multicolumn{4}{|c|}{ Adjuvant therapy } \\
\hline Yes & $28(37.8)$ & $73(35.4)$ & 0.016 \\
\hline No & $46(62.2)$ & $59(64.6)$ & \\
\hline \multicolumn{4}{|l|}{${ }^{\dagger} \mathrm{CEA}(\mathrm{ng} / \mathrm{ml})$} \\
\hline$>5$ & $33(44.6)$ & $56(42.4)$ & 0.763 \\
\hline$\leq 5$ & $41(55.4)$ & $76(57.6)$ & \\
\hline \multicolumn{4}{|c|}{ Pathological stage } \\
\hline Stages I-II & $42(56.8)$ & $67(50.8)$ & 0.408 \\
\hline Stages III-IV & $32(43.2)$ & $65(49.2)$ & \\
\hline \multicolumn{4}{|c|}{ Differentiated degree } \\
\hline High-middle & $61(82.4)$ & $112(84.8)$ & 0.650 \\
\hline Minor & $13(17.6)$ & $20(15.2)$ & \\
\hline \multicolumn{4}{|c|}{ Lymphatic invasion } \\
\hline Yes & $32(43.2)$ & $67(50.8)$ & 0.300 \\
\hline No & $42(56.8)$ & $65(49.2)$ & \\
\hline \multicolumn{4}{|c|}{ Vascular invasion } \\
\hline Yes & $27(36.5)$ & $72(54.5)$ & 0.013 \\
\hline No & $47(63.5)$ & $60(45.5)$ & \\
\hline
\end{tabular}

${ }^{\dagger}$ KPS: $\triangle$ SII: the change in the systemic immune-inflammation index; KPS: Karnofsky Performance Status; CEA: carcinoembryonic antigen.

95\% $\mathrm{CI}=1.159-3.227, \quad \mathrm{p}=0.012), \quad$ vascular invasion $(\mathrm{HR}=3.508,95 \% \mathrm{CI}=1.771-5.279, p<0.001)$, and the $\Delta \mathrm{SII}$ $(\mathrm{HR}=4.281,95 \% \mathrm{CI}=2.553-7.719, p<0.001)$ were associated with poor OS (Table 3$)$. Adjuvant therapy $(\mathrm{HR}=0.627$, 95\% CI $=0.395-0.993, p=0.047)$, pathological stages III-IV $(\mathrm{HR}=0.512,95 \% \quad \mathrm{CI}=0.321-0.814, p=0.005)$, vascular invasion $(\mathrm{HR}=3.572,95 \% \mathrm{CI}=2.118-6.024, \mathrm{p}<0.001)$, and the $\Delta \mathrm{SII}(\mathrm{HR}=4.041,95 \% \mathrm{CI}=2.514-6.496, \mathrm{p}<0.001)$ were able to predict DFS (Table 4 ). The multivariate analysis revealed that pathological stages III-IV $(\mathrm{HR}=0.442,95 \%$
$\mathrm{CI}=0.236-0.827, p=0.011)$, vascular invasion $(\mathrm{HR}=2.182$, $95 \% \mathrm{CI}=1.243-3.829, p=0.007)$, and the $\Delta \mathrm{SII}(\mathrm{HR}=4.301$, $95 \% \mathrm{CI}=2.517-7.350, \mathrm{p}<0.001)$ were independent predictors of OS (Table 3). Adjuvant therapy $(\mathrm{HR}=0.415,95 \% \mathrm{CI}=$ $0.250-0.687, p=0.001)$, vascular invasion $(\mathrm{HR}=3.305,95 \%$ $\mathrm{CI}=1.944-5.620, p<0.001)$, and the $\Delta \mathrm{SII}(\mathrm{HR}=4.924,95 \%$ $\mathrm{CI}=2.992-8.102, p<0.001)$ were significant prognostic factors for DFS (Table 4).

\section{Discussion}

In this study, we investigated the association between the $\Delta$ SII and the clinical outcome of CRC. Our study demonstrated that the $\Delta$ SII had an independent prognostic value in CRC patients who underwent curative resection. Our study suggested that the $\Delta$ SII could independently predict survival in colorectal cancer after curative resection.

Some inflammatory index, such as the NLR, LMR, and PLR, have been indicated to be valid prognosticators for CRC [17, 25-27]. However, these prognosticators are typically based on two immunoinflammation cell types, and their predictive reliability for clinical outcomes was limited. The SII has been widely investigated and was demonstrated to be an effective predictor of the prognosis of various malignant tumors [18-20, 28, 29]. Hu et al. [19] initially revealed the prognostic value of the SII in liver cancer and demonstrated that higher preoperative SII heralded shorter survival times. Tong et al. [28] also demonstrated that a high SII was associated with poor outcomes in non-small-cell lung cancer. Jiang et al. [29] conducted a propensity score-matched analysis, which showed that the SII could predict OS in nasopharyngeal carcinoma patients independently.

However, most previous studies only indicated the preoperative value and the results were not consistent. Some studies indicate that SII was proposed as a significant prognostic factor in CRC $[21,22,30]$, whereas other studies were not significant $[23,31]$. Few studies focused on the changes of inflammatory markers before and after treatment, which might reflect the correlation between the host's inherent inflammatory state and immune response. Wang et al. [24] reported that dynamic changes in the SII represent new prognosis indicators for liver cancer that received surgery. As far as we know, this present study was the first to explore the role of the $\Delta$ SII in CRC. These results revealed that the $\Delta$ SII $(p<0.001)$ was an independent risk factor that predicted OS. Furthermore, we confirmed the significant predictive value of the $\Delta$ SII $(p<0.001)$ for DFS. We also observed that the surgical approach, CEA level, differentiation degree, and lymphatic invasion were not significantly associated with OS and DFS for CRC patients. Adjuvant therapy was only associated with DFS. These results revealed that the $\Delta$ SII reflects the systemic immune-inflammatory changes before and after surgery, which may indicate a more accurate prognosis.

The underlying mechanism by which $\Delta$ SII predicts the survival of CRC may be related to platelets, neutrophils, and lymphocytes. Elevated platelet counts can protect circulating tumor cells (CTCs) and promote metastasis by 


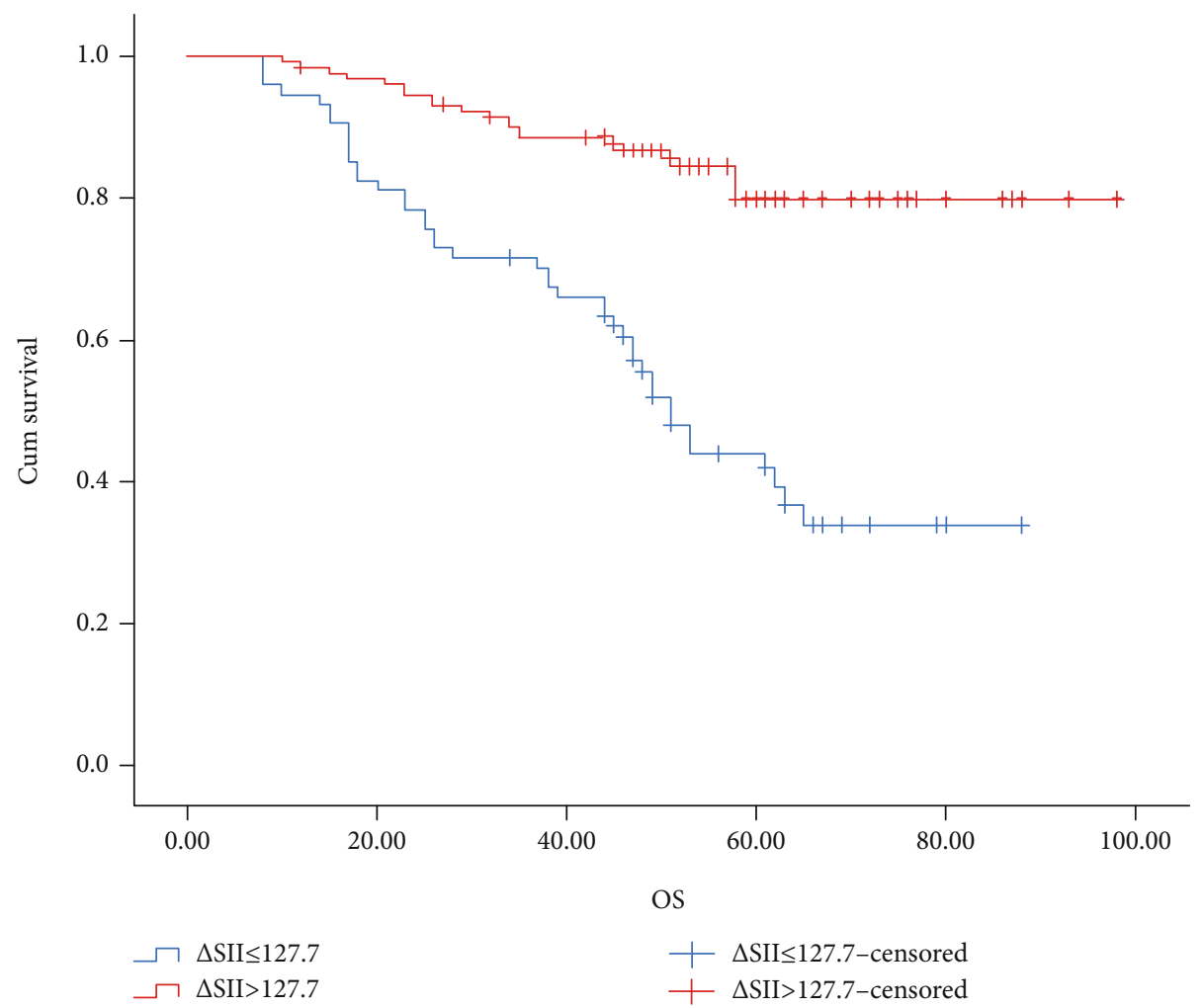

FIGURE 2: Kaplan-Meier graphs of overall survival for patients in the high- $\Delta$ SII group $(>127.7)$ and low- $\Delta$ SII group ( $\leq 127.7)$.

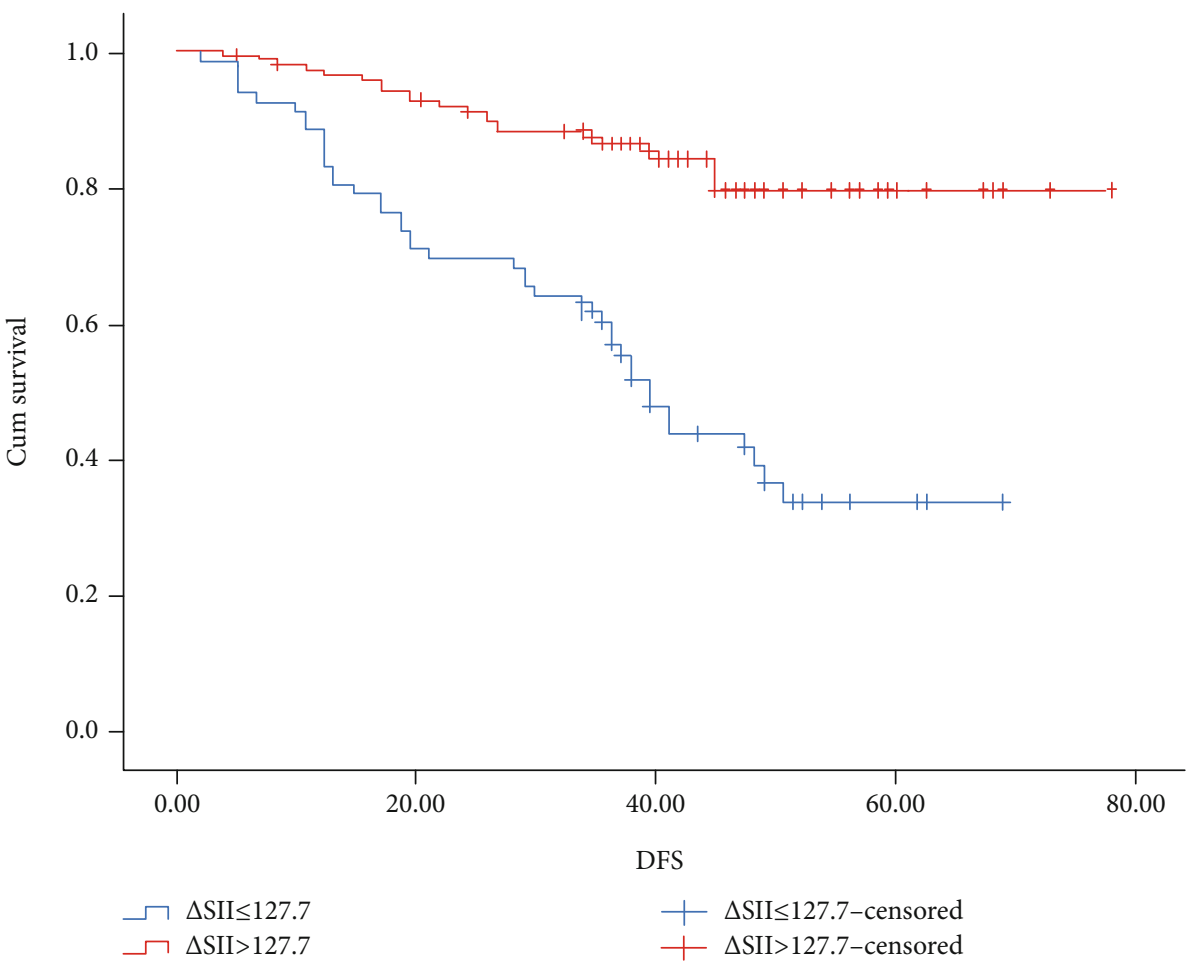

FIGURE 3: Kaplan-Meier graphs of disease-free survival for patients in the high- $\Delta$ SII group (>127.7) and low- $\Delta$ SII group ( $\leq 127.7)$.

inducing CTC epithelial-mesenchymal transition [32, 33]. Neutrophils have been reported to have the potential to promote tumor progression by establishing a tumor microenvi- ronment, including a lot of inflammatory index, such as growth factors (CXCL8), proangiogenic factors (VEGF), and antiapoptotic factors (NF- $\kappa \mathrm{B}$ ) [34-36]. Lymphocytes 
TABLE 3: Univariable and multivariable analysis for overall survival.

\begin{tabular}{|c|c|c|c|c|c|c|}
\hline \multirow{2}{*}{ Variables } & \multicolumn{3}{|c|}{ Univariable analysis } & \multicolumn{3}{|c|}{ Multivariable analysis } \\
\hline & HR & $95 \% \mathrm{CI}$ & $p$ & HR & $95 \% \mathrm{CI}$ & $p$ \\
\hline Age $(\leq 60 />60$, years $)$ & 1.162 & $0.693-1.946$ & 0.569 & & & \\
\hline Gender (male/female) & 1.300 & $0.795-2.126$ & 0.295 & & & \\
\hline${ }^{\dagger} \mathrm{KPS}(>80 / \leq 80)$ & 0.841 & $0.492-1.439$ & 0.527 & & & \\
\hline Smoking status (yes/no) & 1.890 & $1.028-3.476$ & 0.040 & & & \\
\hline Localization (rectum/colon) & 0.947 & $0.575-1.560$ & 0.831 & & & \\
\hline Surgical approach (laparoscopy/open) & 0.985 & $0.601-1.615$ & 0.952 & & & \\
\hline Adjuvant therapy (no/yes) & 0.652 & $0.398-1.069$ & 0.090 & & & \\
\hline${ }^{\dagger} \mathrm{CEA}(\leq 5 />5, \mathrm{ng} / \mathrm{ml})$ & 0.692 & $0.417-1.147$ & 0.153 & & & \\
\hline Pathological stage (I-II/III-IV) & 0.451 & $0.270-0.755$ & 0.002 & 0.442 & $0.236-0.827$ & 0.011 \\
\hline Differentiated degree (high-middle/minor) & 0.476 & $0.262-0.862$ & 0.014 & & & \\
\hline Lymphatic invasion (yes/no) & 1.934 & $1.159-3.227$ & 0.012 & & & \\
\hline Vascular invasion (yes/no) & 3.508 & $1.771-5.279$ & $<0.001$ & 2.182 & $1.243-3.829$ & 0.007 \\
\hline${ }^{\dagger} \Delta$ SII $(>127.7 / \leq 127.7)$ & 4.281 & $2.553-7.719$ & $<0.001$ & 4.301 & $2.517-7.350$ & $<0.001$ \\
\hline
\end{tabular}

${ }^{\dagger} \mathrm{KPS}: \Delta$ SII: the change in the systemic immune-inflammation index; KPS: Karnofsky Performance Status; CEA: carcinoembryonic antigen.

TABLE 4: Univariable and multivariable analysis for disease-free survival.

\begin{tabular}{|c|c|c|c|c|c|c|}
\hline \multirow{2}{*}{ Variables } & \multicolumn{3}{|c|}{ Univariable analysis } & \multicolumn{3}{|c|}{ Multivariable analysis } \\
\hline & HR & $95 \% \mathrm{CI}$ & $p$ & HR & $95 \% \mathrm{CI}$ & $p$ \\
\hline Age $(\leq 60 />60$, years $)$ & 1.100 & $0.682-1.773$ & 0.696 & & & \\
\hline Gender (male/female) & 1.342 & $0.850-2.119$ & 0.207 & & & \\
\hline${ }^{\dagger} \mathrm{KPS}(>80 / \leq 80)$ & 0.785 & $0.477-1.293$ & 0.343 & & & \\
\hline Smoking status (yes/no) & 1.401 & $0.832-2.361$ & 0.205 & & & \\
\hline Localization (rectum/colon) & 0.824 & $0.515-1.318$ & 0.420 & & & \\
\hline Surgical approach (laparoscopy/open) & 0.929 & $0.588-1.470$ & 0.754 & & & \\
\hline Adjuvant therapy (no/yes) & 0.627 & $0.395-0.993$ & 0.047 & 0.415 & $0.250-0.687$ & 0.001 \\
\hline${ }^{\dagger} \mathrm{CEA}(\leq 5 />5, \mathrm{ng} / \mathrm{ml})$ & 0.818 & $0.514-1.302$ & 0.396 & & & \\
\hline Pathological stage (I-II/III-IV) & 0.512 & $0.321-0.814$ & 0.005 & & & \\
\hline Differentiated degree (high-middle/minor) & 0.624 & $0.349-1.118$ & 0.113 & & & \\
\hline Lymphatic invasion (yes/no) & 1.583 & $0.994-2.521$ & 0.053 & & & \\
\hline Vascular invasion (yes/no) & 3.572 & $2.118-6.024$ & $<0.001$ & 3.305 & $1.944-5.620$ & $<0.001$ \\
\hline${ }^{\dagger} \Delta \mathrm{SII}(>127.7 / \leq 127.7)$ & 4.041 & $2.514-6.496$ & $<0.001$ & 4.924 & $2.992-8.102$ & $<0.001$ \\
\hline
\end{tabular}

${ }^{\dagger}$ KPS: $\Delta$ SII: the change in the systemic immune-inflammation index; KPS: Karnofsky Performance Status; CEA: carcinoembryonic antigen.

can inhibit tumor cell proliferation and migration by secreting cytokines, inducing cytotoxic cell death [37]. Therefore, a higher SII indicates a stronger inflammatory reaction while weaker immune defense, leading to poor clinical outcomes.

There are several limitations of this study. Firstly, the current study used a retrospective design with a small population. Although we utilized data that was obtained from the electronic medical records, biases such as selection bias may exist. Secondly, the impact of treatment after recurrence on survival has not been analyzed due to lack of sufficient information. Therefore, further studies with larger sample sizes and prospective research designs are necessary to demonstrate the relationship between the $\Delta$ SII and prognosis for CRC patients.

In conclusion, our study demonstrated $\Delta$ SII was an independent prognostic factor for CRC patients undergo- ing curative resection. The patients with CRC would receive more individualized treatment according to the $\Delta$ SII in the future.

\section{Data Availability}

The datasets generated during and analyzed during the current study are available from the corresponding author on reasonable request.

\section{Conflicts of Interest}

The authors declared that no conflicts of interest exist with respect to the authorship and/or publication of this article. 


\section{Authors' Contributions}

Qingqing Chen, Haohao $\mathrm{Wu}$, and Xinwei Guo contributed equally to this work.

\section{Acknowledgments}

This study was supported by Jiangsu Provincial Medical Youth Talent (QNRC2016234); the Gusu Health Talent Program (2020117); High-level Health Talent "Six One Project" Top Talent Program of Jiangsu Province (LGY2018009); and Suzhou Cancer Clinical Medical Center (Szzx201506).

\section{References}

[1] J. Ferlay, I. Soerjomataram, R. Dikshit et al., "Cancer incidence and mortality worldwide: sources, methods and major patterns in GLOBOCAN 2012," International Journal of Cancer, vol. 136, no. 5, pp. E359-E386, 2015.

[2] W. Chen, R. Zheng, P. D. Baade et al., "Cancer statistics in China, 2015," CA: A Cancer Journal for Clinicians, vol. 66, no. 2, pp. 115-132, 2016.

[3] M. X. Li, X. M. Liu, X. F. Zhang et al., "Prognostic role of neutrophil-to-lymphocyte ratio in colorectal cancer: a systematic review and meta-analysis," International Journal of Cancer, vol. 134, no. 10, pp. 2403-2413, 2014.

[4] Z. Mei, Y. Liu, C. Liu et al., “Tumour-infiltrating inflammation and prognosis in colorectal cancer: systematic review and meta-analysis," British Journal of Cancer, vol. 110, no. 6, pp. 1595-1605, 2014.

[5] S. M. Bentzen, I. Balslev, M. Pedersen et al., "Time to locoregional recurrence after resection of Dukes' B and C colorectal cancer with or without adjuvant postoperative radiotherapy. A multivariate regression analysis," British Journal of Cancer, vol. 65, no. 1, pp. 102-107, 1992.

[6] D. M. Hari, A. M. Leung, J. H. Lee et al., “AJCC Cancer staging manual 7th edition criteria for colon cancer: do the complex modifications improve prognostic assessment?," Journal of the American College of Surgeons, vol. 217, no. 2, pp. 181190, 2013.

[7] F. Mehrkhani, S. Nasiri, K. Donboli, A. Meysamie, and A. Hedayat, "Prognostic factors in survival of colorectal cancer patients after surgery," Colorectal Disease, vol. 11, no. 2, pp. 157-161, 2009.

[8] I. Zlobec and A. Lugli, "Prognostic and predictive factors in colorectal cancer," Journal of Clinical Pathology, vol. 61, no. 5, pp. 561-569, 2008.

[9] G. J. Guthrie, K. A. Charles, C. S. Roxburgh, P. G. Horgan, D. C. McMillan, and S. J. Clarke, "The systemic inflammation-based neutrophil-lymphocyte ratio: experience in patients with cancer," Critical Reviews in Oncology/Hematology, vol. 88, no. 1, pp. 218-230, 2013.

[10] D. C. McMillan, "The systemic inflammation-based Glasgow Prognostic Score: a decade of experience in patients with cancer," Cancer Treatment Reviews, vol. 39, no. 5, pp. 534-540, 2013.

[11] M. Hernandez, R. Martin, M. D. Garcia-Cubillas, P. MaesoHernandez, and M. L. Nieto, "Secreted PLA2 induces proliferation in astrocytoma through the EGF receptor: another inflammation-cancer link," Neuro-Oncology, vol. 12, no. 10, pp. 1014-1023, 2010.
[12] A. V. Nguyen, Y. Y. Wu, and E. Y. Lin, "STAT3 and sphingosine-1-phosphate in inflammation-associated colorectal cancer," World Journal of Gastroenterology, vol. 20, no. 30, pp. 10279-10287, 2014.

[13] A. V. Nguyen, Y. Y. Wu, Q. Liu et al., "STAT3 in epithelial cells regulates inflammation and tumor progression to malignant state in colon," Neoplasia, vol. 15, no. 9, pp. 998-1008, 2013.

[14] L. Borsig, M. J. Wolf, M. Roblek, A. Lorentzen, and M. Heikenwalder, "Inflammatory chemokines and metastasis-tracing the accessory," Oncogene, vol. 33, no. 25, pp. 3217-3224, 2014.

[15] C. I. Diakos, K. A. Charles, D. C. McMillan, and S. J. Clarke, "Cancer-related inflammation and treatment effectiveness," The Lancet Oncology, vol. 15, no. 11, pp. e493-e503, 2014.

[16] H. Zhang, X.-W. Guo, X.-X. Yin, Y.-C. Liu, and S.-J. Ji, "Nomogram-integrated C-reactive protein/albumin ratio predicts efficacy and prognosis in patients with thoracic esophageal squamous cell carcinoma receiving chemoradiotherapy," Cancer Management and Research, vol. 11, pp. 9459-9468, 2019.

[17] Z.-Y. Zou, H.-L. Liu, N. Ning, S.-Y. Li, X.-H. Du, and R. Li, "Clinical significance of pre-operative neutrophil lymphocyte ratio and platelet lymphocyte ratio as prognostic factors for patients with colorectal cancer," Oncology Letters, vol. 11, no. 3, pp. 2241-2248, 2016.

[18] D. Guo, J. Zhang, W. Jing et al., "Prognostic value of systemic immune-inflammation index in patients with advanced nonsmall-cell lung cancer," Future Oncology, vol. 14, no. 25, pp. 2643-2650, 2018.

[19] B. Hu, X. R. Yang, Y. Xu et al., "Systemic immuneinflammation index predicts prognosis of patients after curative resection for hepatocellular carcinoma," Clinical Cancer Research, vol. 20, no. 23, pp. 6212-6222, 2014.

[20] C. Lolli, U. Basso, L. Derosa et al., "Systemic immuneinflammation index predicts the clinical outcome in patients with metastatic renal cell cancer treated with sunitinib," Oncotarget, vol. 7, no. 34, pp. 54564-54571, 2016.

[21] Q. K. Xie, P. Chen, W. M. Hu et al., "The systemic immuneinflammation index is an independent predictor of survival for metastatic colorectal cancer and its association with the lymphocytic response to the tumor," Journal of Translational Medicine, vol. 16, no. 1, p. 273, 2018.

[22] J. Yang, H. Xu, X. Guo et al., "Pretreatment inflammatory indexes as prognostic predictors for survival in colorectal cancer patients receiving neoadjuvant chemoradiotherapy," Scientific Reports, vol. 8, no. 1, p. 3044, 2018.

[23] Y. Y. Wang, Z. Z. Liu, D. Xu, M. Liu, K. Wang, and B. C. Xing, "Fibrinogen Albumin Ratio Index (FARI): a more promising inflammation-based prognostic marker for patients undergoing hepatectomy for colorectal liver metastases," Annals of Surgical Oncology, vol. 26, no. 11, pp. 36823692, 2019.

[24] B. L. Wang, L. Tian, X. H. Gao et al., "Dynamic change of the systemic immune inflammation index predicts the prognosis of patients with hepatocellular carcinoma after curative resection," Clinical Chemistry and Laboratory Medicine, vol. 54, no. 12, pp. 1963-1969, 2016.

[25] D. Guo, A. Han, W. Jing et al., "Preoperative to postoperative change in neutrophil-to-lymphocyte ratio predict survival in colorectal cancer patients," Future Oncology, vol. 14, no. 12, pp. 1187-1196, 2018. 
[26] J. H. Kim, J. Y. Lee, H. K. Kim et al., "Prognostic significance of the neutrophil-to-lymphocyte ratio and platelet-tolymphocyte ratio in patients with stage III and IV colorectal cancer," World Journal of Gastroenterology, vol. 23, no. 3, pp. 505-515, 2017.

[27] J. Peng, H. Li, Q. Ou et al., "Preoperative lymphocyte-tomonocyte ratio represents a superior predictor compared with neutrophil-to-lymphocyte and platelet-to-lymphocyte ratios for colorectal liver-only metastases survival," Oncotargets and Therapy, vol. 10, pp. 3789-3799, 2017.

[28] Y. S. Tong, J. Tan, X. L. Zhou, Y. Q. Song, and Y. J. Song, "Systemic immune-inflammation index predicting chemoradiation resistance and poor outcome in patients with stage III non-small cell lung cancer," Journal of Translational Medicine, vol. 15, no. 1, p. 221, 2017.

[29] W. Jiang, Y. Chen, J. Huang et al., "Systemic immuneinflammation index predicts the clinical outcome in patients with nasopharyngeal carcinoma: a propensity score-matched analysis," Oncotarget, vol. 8, no. 39, pp. 66075-66086, 2017.

[30] M. Dong, Y. Shi, J. Yang et al., "Prognostic and clinicopathological significance of systemic immune-inflammation index in colorectal cancer: a meta-analysis," Therapeutic Advances in Medical Oncology, vol. 12, p. 175883592093742, 2020.

[31] Z. Q. Zhou, S. Pang, X. C. Yu et al., "Predictive values of postoperative and dynamic changes of inflammation indexes in survival of patients with resected colorectal cancer," Current Medical Science, vol. 38, no. 5, pp. 798-808, 2018.

[32] M. Labelle, S. Begum, and R. O. Hynes, "Direct signaling between platelets and cancer cells induces an epithelialmesenchymal-like transition and promotes metastasis," Cancer Cell, vol. 20, no. 5, pp. 576-590, 2011.

[33] D. Schumacher, B. Strilic, K. K. Sivaraj, N. Wettschureck, and S. Offermanns, "Platelet-derived nucleotides promote tumorcell transendothelial migration and metastasis via $\mathrm{P} 2 \mathrm{Y} 2$ receptor," Cancer Cell, vol. 24, no. 1, pp. 130-137, 2013.

[34] A. Paramanathan, A. Saxena, and D. L. Morris, "A systematic review and meta-analysis on the impact of pre-operative neutrophil lymphocyte ratio on long term outcomes after curative intent resection of solid tumours," Surgical Oncology, vol. 23, no. 1, pp. 31-39, 2014.

[35] K. A. Scilla, S. M. Bentzen, V. K. Lam et al., "Neutrophil-lymphocyte ratio is a prognostic marker in patients with locally advanced (stage IIIA and IIIB) non-small cell lung cancer treated with combined modality therapy," The Oncologist, vol. 22, no. 6, pp. 737-742, 2017.

[36] X. Zhao, D.-M. Zhu, W.-J. Gan et al., "Lentivirus-mediated shRNA interference targeting vascular endothelial growth factor inhibits angiogenesis and progression of human pancreatic carcinoma," Oncology Reports, vol. 29, no. 3, pp. 1019-1026, 2013.

[37] C. Ferrone and G. Dranoff, "Dual roles for immunity in gastrointestinal cancers," Journal of Clinical Oncology, vol. 28, no. 26, pp. 4045-4051, 2010. 